\section{PS-144 PERFORMANCE OF CLINICAL PREDICTION RULES IN YOUNG FEBRILE INFANTS AT THE EMERGENCY DEPARTMENT}

${ }^{1}$ E Kerkhof, ${ }^{2} \mathrm{~B}$ Gomez, ${ }^{3} Y$ Vergouwe, ${ }^{2} \mathrm{~S}$ Mintegi, ${ }^{1} \mathrm{HA}$ Moll, ${ }^{1} \mathrm{R}$ Oostenbrink. ${ }^{1}$ General Paediatrics, Erasmus Medical Center-Sophia Children's Hospital, Rotterdam, Netherlands; ${ }^{2}$ Pediatric Emergency Department, Cruces University Hospital, Bilbao, Spain; ${ }^{3}$ Department of Public Health Clinical Decision Making, Erasmus Medical Center, Rotterdam, Netherlands

\subsection{6/archdischild-2014-307384.439}

Background Clinical prediction rules (CPRs) are developed to aid the identification of serious infections (SI), but their value in young febrile infants remains unclear.

Aim To systematically review existing CPRs and subsequently validate these CPRs in two external cohorts of young febrile infants at risk for SI in the Netherlands ${ }^{1}(\mathrm{~N}=925$; $\leq 1$ year $)$ and $\operatorname{Spain}^{2}$ ( $\mathrm{N}=2148 ; \leq 3$ months).

Methods We included seven multivariable developed CPRs for febrile children to predict SI, including clinical predictors and/or diagnostic tests results. CPR performance was assessed by sensitivity, specificity, calibration analyses and area under the receiver operating characteristic curve (AUC).

Results All CPRs (including 19 different predictors) originally performed moderate-good (AUC0.60-0.93). The original cohorts, with SI prevalence variation of $0.8-27 \%$, varied between 381 and 5279 febrile children. Almost all CPRs were derived in emergency care populations including wide age ranges of $0-16$ years.

Validation of CPRs missing $\geq 2 / 3$ of the required variables was not performed, resulting in limited evaluation of two CPRs including eg capillary refill time and vital signs (heart/respiratory rate) in the Spanish cohort.

Four out of 7 CPRs showed acceptable ROC-areas (0.760.89 ) in both cohorts. Sensitivities of CPRs predicting high/low risks ranged from $0.60-0.93$ and specificities from 0.71-0.97. Three CPRs were non-informative (AUC 0.49-0.53). Calibration slopes were mostly $<1$, which could indicate overestimation of predictor effects in young febrile infants.

Discussion and Conclusion Four (out of 7) CPRs showed comparable performance in the identification of SI in infants $\leq 1$ year, although with more emphasise on their rule-in value (specificity). However, predictor effects were generally overestimated.

\section{PS-145 PRETERM INFANTS TRANSPORTATION BETWEEN TERTIARY CARE CENTRES (TCC) WITHIN FIRST HOURS OF LIFE: RESTROSPECTIVE COHORT STUDY}

${ }^{1} \mathrm{G}$ Jourdain, ${ }^{2} \mathrm{~F}$ Longhini, ${ }^{1} \mathrm{P}$ Quentin, ${ }^{2} \mathrm{~A}$ Boët, ${ }^{3} \mathrm{~L}$ Julé, ${ }^{1} \mathrm{~F}$ Ammar, ${ }^{1} \mathrm{JL}$ Chabernaud, ${ }^{2} \mathrm{D}$ De Luca. ${ }^{1}$ Division of Pediatrics and Neonatal Critical Care and Mobile Intensive Care Unit, South Paris University Hospitals Medical Center "A. Beclere" Clamart France, Clamart, France; ${ }^{2}$ Division of Pediatrics and Neonatal Critical Care, South Paris University Hospitals Medical Center "A. Beclere" Clamart France, Clamart, France; ${ }^{3}$ Division of Pediatrics and Neonatal Critical Care, South Paris University Hospitals Medical Center "Kremlin Bicêtre" France, Kremlin Bicêtre, France

\subsection{6/archdischild-2014-307384.440}

Introduction Regionalization in perinatal care improved neonatal survival for 2 decades. Perinatal transport is known to be a bad prognostic factor for preterm neonates born in second level centres. No data exist for babies born in TCC who had to be transferred to other TTC. We evaluate short term clinical outcomes of preterm infants transferred between TCC.

\section{Abstract PS-145 Table 1}

\begin{tabular}{|l|l|l|l|l|}
\hline Clinical features & Cases & Controls & $\begin{array}{l}\text { OR for } \\
\text { transfer }\end{array}$ & P value \\
\hline RDS & $35(56.5 \%)$ & $33(53.2 \%)$ & $1.1(0.5-2.3)$ & 0.718 \\
\hline IRRF & $4(6.5 \%)$ & $5(8.1 \%)$ & $0.8(0.2-3.1)$ & 0.730 \\
\hline Air leaks & $2(3.2 \%)$ & $1(1.6 \%)$ & $2.0(0.1-23.0)$ & 0.567 \\
\hline Hypotension & $1(1.6 \%)$ & $1(1.6 \%)$ & $1.0(0.1-16.0)$ & 0.999 \\
\hline Outcomes & Cases & Controls & $\begin{array}{l}\text { OR for } \\
\text { transfer }\end{array}$ & P value \\
\hline IVH $>2$ & $3(4.5 \%)$ & $2(3.2 \%)$ & $1.5(0.2-9.4)$ & 0.650 \\
\hline PVL & $5(8.1 \%)$ & $4(6.5 \%)$ & $1.3(0.3-5.0)$ & 0.730 \\
\hline NEC & $1(1.6 \%)$ & $1(1.6 \%)$ & $1.0(0.1-16.0)$ & 0.999 \\
\hline BPD & $6(9.7 \%)$ & $5(8.1 \%)$ & $1.2(0.3-4.2)$ & 0.752 \\
\hline & Cases & Controls & $\beta$ for transfer & P value \\
\hline $\begin{array}{l}\text { NICU Stay } \\
\text { (days) }\end{array}$ & $8[4-34]$ & $9[5.8-16]$ & $6.4(-1.3-14)$ & 0.103 \\
\hline
\end{tabular}

Methods We retrospectively analysed all neonates aged $\leq 32$ weeks gestation transferred before $6 \mathrm{~h}$ of life from the South Paris University Hospitals to another TCC. Transfer was due to organisational problems. Control group consisted of neonates born the month before or after the cases and matched for gestational age, birth weight and CRIB-II. Simple linear and logistic regressions were used for analysis.

Results We included 60 cases and 60 controls. The two groups were similar for basic clinical characteristics. No difference in clinical features (RDS, infection related respiratory failure, air leaks, hypotension) were present between the groups (Table 1). Early outcomes (IVH, periventricular leucomalacy, NEC, BPD and NICU stay) rates were not influenced by the transfer transport ((Table 1$)$

Conclusions Perinatal transfer for preterm babies born in a TCC is not a negative prognostic factor. It is conceivable that optimal care in delivery room is a keystone for better outcome.

\section{PS-146 THE LOW RISK ANKLE RULE CAN SAFELY BE APPLIED TO A UK PAEDIATRIC POPULATION}

${ }^{1} \mathrm{~N}$ Lane, ${ }^{2} \mathrm{H}$ Yorke, ${ }^{2} \mathrm{C}$ Stewart. ${ }^{1}$ School of Medicine, Imperial College London, London, UK; ${ }^{2}$ Paediatric Department, Chelsea and Westminster Hospital, London, UK

\subsection{6/archdischild-2014-307384.441}

Background and aims Ankle injuries (AI) are a common presentation to Paediatric Emergency Departments (PED). The Low Risk Ankle Rule (LRAR) is a validated clinical decision to determine grounds for radiography. It identifies swelling and tenderness isolated to the distal fibula and adjacent lateral ligaments distal to the anterior tibial joint line as low-risk; where ankle radiography $(\mathrm{aXR})$ is not necessary to further exclude injury. The primary outcome is to evaluate LRAR in PED; with secondary aim to determine potential cost-savings.

Method LRAR was applied retrospectively to all paediatric aXR performed over 6-months (1/04/13-1/10/13) following presentation to PED with an AI. Data was accessed using Electronic Patient Records.

Results 311 aXR were performed of which 175 had sufficient data to be included in the study. Applying LRAR, 110 children fit the low-risk group (LRG.) Thus 110 unnecessary aXR were performed (62.86\% reduction,) producing savings of $£ 1,650$. Within the LRG 8 fractures were confirmed but required no treatment beyond short-term below knee Plaster-Of-Paris, Aircast ${ }^{\circledR}$ boot or equivalent. 3 received no treatment or follow-up. There were 26 fracture clinic referrals from the LRG. All 21 non-fractures were discharged at initial follow-up. The confirmed fractures were all discharged by second follow-up. 
Applying LRAR would have reduced follow-up resulting in costsavings of $£ 8,068$; in total $£ 9,718$.

Conclusion Implementing the LRAR in children presenting with AI in the UK is safe from a clinical view point; will reduce radiography and follow-up, resulting in significant cost-savings.

\section{PS-147 ULTRASOUND CARDIAC OUTPUT MONITORING (USCOM) IN MECHANICALLY VENTILATED CRITICALLY ILL CHILDREN}

J Fremuth, J Kobr, K Pizingerova, L Sasek, J Zamboryova. Pediatrics, Medical Faculty Hospital Plzen Charles University Prague, Plzen, Czech Republic

\subsection{6/archdischild-2014-307384.442}

Background and aims Haemodynamic monitoring plays an important role in the management of critically ill patients. The goal of this study was to evaluate haemodynamic changes within first $48 \mathrm{~h}$ after initiation of mechanical ventilation (MV).

Methods Critically ill children were included and divided into two groups according to the indication for MV. Group A ventilated for pulmonary pathology $(\mathrm{P})$, group B ventilated for non pulmonary pathology (NP). Noninvasive haemodynamic monitoring (USCOM) was used in both groups after the initiation of MV (Time 1) and at 6, 12 and $48 \mathrm{~h}$ intervals (Time 2, 3, 4). Parameters such as CI, SVRI, SVI, SBP and DBP were analysed. Strategies of protective MV were applied in both groups.

Results Group A included 36 children, mean age 44 months. Group B included 13 children, mean age 58 months. The comparisons within the groups and between the groups are presented in Table 1.

\begin{tabular}{|c|c|c|c|c|c|c|}
\hline & \multicolumn{2}{|c|}{ Pulmonary group - P } & \multicolumn{4}{|c|}{ Non pulmonary group - NP } \\
\hline & Time 1 & Time 4 & comparison & Time 1 & Time 4 & comparison \\
\hline $\mathrm{Cl}$ & $4.30 \pm 1.25$ & $4.09 \pm 1.08$ & NS & $4.33 \pm 1.57$ & $3.59 \pm 1.03$ & NS \\
\hline SVRI & $1193 \pm 592$ & $1321 \pm 557$ & NS & $1050 \pm 193$ & $1607 \pm 570$ & $p<0.005$ \\
\hline SVI & $34 \pm 11$ & $35 \pm 11$ & NS & $36 \pm 12$ & $37 \pm 12$ & NS \\
\hline SBP & $90 \pm 21$ & $102 \pm 25$ & $\mathrm{p}<0.0142$ & $87 \pm 21$ & $102 \pm 19$ & $\mathrm{p}<0.0251$ \\
\hline DBP & $52 \pm 15$ & $56 \pm 17$ & NS & $47 \pm 15$ & $55 \pm 17$ & NS \\
\hline
\end{tabular}

Conclusions SVRI increased during first $48 \mathrm{~h}$ of ventilation in NP group, SBP increased in both groups. No other clinically significant haemodynamic changes in either group were found.

Acknowledgements The work was supported by project PRVOUK P-36.

\section{PS-148 PRE-HOSPITAL TRANSPORT PRACTICES PREVALENT AMONG CHILDREN REQUIRING PICU ADMISSION IN A TERTIARY CARE CENTRE OF A DEVELOPING COUNTRY}

1J Sankar, ${ }^{2} \mathrm{~N}$ Dev, ${ }^{1} \mathrm{~A}$ Singh, ${ }^{1} \mathrm{~N}$ Dubey. ${ }^{1}$ Pediatrics, PGIMER Dr RML Hospital, New Delhi, India; ${ }^{2}$ Medicine, PGIMER Dr RML Hospital, New Delhi, India

\subsection{6/archdischild-2014-307384.443}

Background and aims While specially trained paediatric transport teams are firmly in place in the developed nations for transporting the critically ill to the paediatric intensive care unit (PICU), the same is not true for the resource restricted ones. Although well known, this finding is underreported. The aim of our study was to evaluate the pre-hospital transport practices of those requiring PICU admission and their subsequent clinical course.
Methods We collected information on the pre-hospital transport factors of children requiring PICU admission at presentation to our paediatric emergency department (PED), over a period of 6 months (Jan-Jun 2013) and recorded their outcomes. The study was approved by the IEC.

Results A total of 319 patients presented to the PED during the study period. Fifty four children (17\%) required PICU admission. Majority (60\%) were males. Septic shock (48\%) was the commonest admitting diagnosis. Only 2 patients referred were transported by ambulance (unaccompanied). Majority (35, 65\%) reached the hospital by public transport systems such as auto rickshaw and bus. The median PIM2 probability was 56\%. Of those admitted, $18 \%$ needed mechanical ventilation, and $46 \%$, inotropic support within the first hour. Sixteen children (30\%) died during PICU stay.

Conclusions There is an urgent need to develop and integrate paediatric retrieval teams into the health care system of our country. Special telemedicine facility or call centres could be set up for this purpose so that the information could reach these teams and the patient could be transferred in a timely and appropriate manner to the nearest PICU available.

\section{PS-148a DOES OBSTRUCTIVE SLEEP APNEA CONTRIBUTE TO ELEVATED INTRACRANIAL PRESSURE IN CHILDREN WITH SYNDROMIC CRANIOSYNOSTOSIS? A PROSPECTIVE COHORT STUDY}

${ }^{1} \mathrm{~B}$ Spruijt, ${ }^{2} \mathrm{KFM}$ Joosten, ${ }^{3} \mathrm{C}$ Driessen, ${ }^{4} \mathrm{MLC}$ van Veelen-Vincent, ${ }^{5} \mathrm{~N}$ Naus, ${ }^{6} \mathrm{D}$ Rizopoulos, ${ }^{7}$ RC Tasker, ${ }^{3}$ IMJ Mathijssen. ${ }^{1}$ Pediatrics/Dutch Craniofacial Center, Erasmus University Medical Center - Sophia Children's Hospital, Rotterdam, Netherlands; ${ }^{2}$ Pediatric Intensive Care, Erasmus University Medical Center - Sophia Children's Hospital, Rotterdam, Netherlands; ${ }^{3}$ Plastic Reconstructive and Hand Surgery/Dutch Craniofacial Center, Erasmus University Medical Center - Sophia Children's Hospital, Rotterdam, Netherlands; ${ }^{4}$ Neurosurgery, Erasmus University Medical Center - Sophia Children's Hospital, Rotterdam, Netherlands; ${ }^{5}$ Ophthalmology, Erasmus University Medical Center Sophia Children's Hospital, Rotterdam, Netherlands; ${ }^{6}$ Biostatistics, Erasmus University Medical Center, Rotterdam, Netherlands; ${ }^{7}$ Neurology and Anaesthesia, Children's Hospital Boston Harvard Medical School, Boston, USA

\subsection{6/archdischild-2014-307384.444}

Background and aims Children with syndromic or complex craniosynostosis have a prevalence of $68 \%$ of obstructive sleep apnea (OSA), which has been associated with an increased risk for developing elevated intracranial pressure (ICP). The objective of this study was to evaluate how often and to what extend OSA increases the risk of elevated ICP in patients with syndromic and complex craniosynostosis and to prospectively evaluate our current clinical treatment protocol.

Methods A prospective observational cohort study of patients with syndromic or complex craniosynostosis treated at the Sophia Children's Hospital, started in January 1st 2007. All patients received repeated sleep studies and fundoscopy (to evaluate papilledema as proxy for elevated ICP), according to a standardised protocol.

Results Sixty-two patients underwent full analysis, with a mean age at time of latest follow-up of 6.0 years. Mean age at first presentation of papilledema was 1.9 years (range 0.4-6.0). Twenty-three of 62 patients (37.1\%) had papilledema, of whom $13(21.0 \%)$ pre-operative. Thirty-nine of 62 (62.9\%) patients had OSA. Compared to patients without OSA, papilledema was not more frequently present in patients with mild or moderate OSA. However, patients with severe OSA had pre-operatively significantly more often papilledema $(p=0.015)$. 